\title{
Small Lung Nodules Detection based on Fuzzy-Logic and Probabilistic Neural Network with Bio-inspired Reinforcement Learning
}

\author{
Giacomo Capizzi (D), Grazia Lo Sciuto (D, Christian Napoli (D), Dawid Połap (D), Marcin Woźniak (iD)
}

\begin{abstract}
Internal organs, like lungs, are very often examined by the use of screening methods. For this purpose we present an evaluation model based on a composition of fuzzy system combined with a neural network. The input image is evaluated by means of custom rules which use type-1 fuzzy membership functions. The results are forwarded to a neural network for final evaluation. Our model was validated by using $X$ ray images with lung nodules. The results shows the high performances of our approach with sensitivity and specificity reaching almost $95 \%$ and $90 \%$ respectively, with an accuracy of $92.56 \%$. The new methodology lower considerably the computational demands and increases detection performances.
\end{abstract}

Index Terms-Chest X-ray screening, Biomedical Image Processing, Automatic Pathology Recognition, Fuzzy-Logic, Probabilistic Neural Network.

\section{INTRODUCTION}

$\mathbf{L}$ UNG diseases are very often examined by using of screening methods. The better is the examination the higher are the chances to help patients in time and save their lifes. Lung cancer is one of the most dangerous diseases, therefore a rapid identification of nodules in the early stadium can reduce deaths. However in-depth analysis of diagnostic images requires large experience and skills. Nowadays due to the recent advancements of clinical and medial sciences, the clinicians dispose of many non-invasive techniques for oncological assessment and diagnosis that are generally preferred to potentially dangerous and more radical approaches (e.g. exploration surgery).

The imaging diagnostic tools for medicinal applications are essentially founded on ecography, x-rays, magnetic resonance imaging (MRI), computer tomography scan (CTS), and positron emission tomography (PET). X-ray imagery is a

G. Capizzi is with the Department of Electrical, Electronics and Informatics Engineering, University of Catania, Viale Andrea Doria 6, 95125 Catania, ITALY and also with the Faculty of Applied Mathematics, Silesian University of Technology, Kaszubska 23, 44-100 Gliwice, POLAND e-mail: gcapizzi@dees.unict.it.

G. Lo Sciuto is with the Department of Electrical, Electronics and Informatics Engineering, University of Catania, Viale Andrea Doria 6, 95125 Catania, ITALY.

C. Napoli is with the Department of Computer, Control, and Management Engineering "Antonio Ruberti", Sapienza University of Rome, Via Ariosto, 25, 00185 Roma, ITALY.

D. Połap and M. Woźniak are with the Faculty of Applied Mathematics, Silesian University of Technology, Kaszubska 23, 44-100 Gliwice, POLAND e-mail: Dawid.Polap@polsl.pl, Marcin.Wozniak@polsl.pl.

Manuscript received DD Month, 2019; revised DD Month, 2019. paramount exam for a correct diagnosis of potentially cancerous lung nodules. Such a diagnostic method is fundamental for radiologists and clinicians in order to select a correct treatment process.

The examination of $\mathrm{x}$-ray images is very important for many lung disorders and diseases even in an early stage, ie. we can detect pre symptomatic changes in the structure of lung tissues. For this reason it is important to be able to dispose of an efficient methodology to evaluate $\mathrm{x}$-ray images, since in general only $\approx 30 \%$ of examinations are done in the respiratory disease early stage. In this stadium the symptoms are not often well visible therefore without a precise methodology a correct diagnosis can be unfortunately omitted. From X-ray images we can evaluate several pathological conditions, and various research present that even $90 \%$ of peripheral neoplasms and $65-70 \%$ of central neoplasms can be efficiently diagnosed from X-ray images in the early stadium of disease. In any case unfortunately the most vulnerable factor is human, since many variables can influence the radiologist ability to detect the illness. The quality of the images can tamper with a clear visibility of all tissues as well as visualize the tissues as opaque or disformed. Sometimes several tissues can be placed in superimpositions with bones which can cover them. Unfortunately, the extreme variability of the matter and the subjective evolution of the illness in each patient can potentially tamper with the correctness of the diagnosis made by a radiologist. Therefore to improve the accuracy of their diagnosis the medical specialists can benefit from intelligent methods of diagnostic support. Moreover automated approaches to the process of analysis bring many advantages for faster and accurate diagnosis.

Intelligent classification systems, and particularly convolutional neural networks (CNNs) and other deep learning techniques can be used as an efficient aid for lung cancer diagnosis and the consequent determination of a therapeutic approach. Among the possible applications of CNNs based classification techniques we can include solid tumors diagnosis and discrimination between malignant cancers with respect to benignant nodules. Deep learning strategies can be partitioned into four classifications: CNN-based methods, restricted Boltzmann machines (RBMs), autoencoders, and Neuro-Fuzzy methods. In this paper we present a new Neuro-Fuzzy methodology for the detection and classification of lungs small nodules by using $\mathrm{x}$ ray images. In each screening the radiation source sends waves 
from $10 \mathrm{pm}$ to $10 \mathrm{~nm}$, which cross the patient body and reach detector. The received waves compose an image of tissues in human body depicted in correspondence to absorption capacity of our organs. Therefore bones and other parts with higher density compose more dens shapes on the x-ray image. In these tissues where density is lower also absorption of the waves is lower, what appears in shapes of darker reflection in the images. In this way in the $\mathrm{x}$-ray image lungs are visible in a form of shaded tissues, but if the wrong happens to lung tissues these will have higher density and therefore higher visibility in the x-ray image. In medical examinations of lung disorders doctors use this and compare tissues in images by this property.

In this work we have used $x$-ray images from participating clinic and open data set radiologykey.com. The dataset is composed by images that come from the open data set radiologykey.com and from clinical trials of nodules in lungs from Zagłębiowskie Oncology Centre, Poland. In Fig. 1 are displayed some samples of lung x-ray images coming from the dataset located at http://radiologykey.com/solitaryand-multiple-pulmonary-nodules.

In this paper we present a novel methodology for the detection of lung carcinomas, based on a Fuzzy logic segmentation method and on a probabilistic neural network. The system has been designed to obtain a an accurate detection of the presence or absence of lung carcinomas. The results show that the proposed approach provides an accuracy of $92.56 \%$, while the sensitivity is $95.56 \%$ and the specificity is $90.79 \%$. The main advantages of the proposed approach are: a good correct detection rate $(92.56 \%)$ and the fact that it can detect lowcontrast nodules with a diameter lower or equal to $20 \mathrm{~mm}$.

\section{A. Related works}

Untreated symptoms of cancer eventually lead to serious consequences and even death in the final stage of the cancer [1], [2]. The research on automated decision support in diagnosis of X-ray images is visible in many aspects. In [3] a support vector machine was using radiomic features to to estimate changes in lungs tissues. In [4] the nodules in lungs were detected by the use of significant features extraction, while in [5] a polygon approximation was implemented for detection of small nodules.

Fuzzy Systems play very important role in medical diagnosis, in facts, mainly due to their intrinsic properties, such approaches have been proven very proficient for feature extraction and image segmentation [6], [7]. In fact over the last two decades, researchers proposed several fuzzy system modeling methods that can extract the hidden rules of a system automatically by using historical data. Conventional Fuzzy system modeling techniques utilize type-1 fuzzy sets in order to capture the uncertainty in the system. However, since type1 fuzzy sets express the belongingness of a crisp value of a base variable in a fuzzy set by a crisp membership value, they cannot fully capture the uncertainties due to imprecision in identifying membership functions. Higher types of fuzzy sets can be a remedy to address this issue. Since the computational complexity of operations on fuzzy sets are increasing with the increasing type of the fuzzy set, the use of type-2 fuzzy sets and linguistic logical connectives drew a considerable amount of attention in the realm of fuzzy system modeling in the last two decades [8].

In this work we branch off the classical use of fuzzy sets as a segmentation tool, in facts, while such approaches are generally used for thresholding [9], [10], in our model we make use of a type-1 fuzzy set to locate and aggregate valid pixels to be further analysed for classification purposes by means of a probabilistic neural network.

The use of the type- 1 fuzzy set, in this work, is due to the fact that for the nodules classification we use a neural network, while the fuzzy logic is aimed only to cluster the possible pixels belonging to lungs nodules, so it is not necessary an high degree of accuracy in the measurement of uncertainty. On the other hand, the use of the type-2 fuzzy set would not lead to an improvement in the performance of the proposed procedure for lung nodules classification, while it would increase the computational burden of the procedure.

Computational intelligence, ie. neural networks are reported as efficient detectors of nodules presence. In [11] a Neural Network classifier was evaluating changes in the texture and shape. In [12], [13] were applied a more complex structures, in particular Convolutional Neural Network and Extreme Learning Machine to extract and detect nodules from X-ray lung images.

\section{Methods}

In this work we used probabilistic neural networks combined with fuzzy logic to design a novel technique for the detection of lung cancers. We used fuzzy logic for the segmentation of chest $\mathrm{x}$-ray imagery for the detection of lung carcinomas. Moreover probabilistic neural networks allowed us to improve the detection accuracy of such a pathology, determining whether or not the patient is developing a malignant cancer.

An X-ray image results from the different shielding attitude of the biological tissues with respect to $\mathrm{x}$ rays. The darkest area identify a small absorption rate by the tissues (e.g. soft tissues, muscles, skin, etc.), on the contrary the lighter areas correspond to the greater absorption rates (e.g. bones, dense tissues). An optical study of the X-ray make it possible to recognize the presence of potentially dangerous tissues due to their augmented density. These tissues appears as lighter areas with respect to the surrounding background. Therefore a lung carcinoma can be identified analyzing the X-ray image, as a gray-levels picture, and looking for local variances.

The proposed technique detects and localize potentially dangerous lung nodules by calculating the local variance on each pixel of the x-ray image. In this manner we obtain a variance matrix in which each cell represent the local variance of each pixel in the original $x$-ray image.

The local variance in a gray level image $I$ is defined as:

$$
\operatorname{var}\left(I_{i, j}\right)=E\left\{\left(I_{i, j}-\bar{I}_{i, j}\right)^{2}\right\}
$$

where $\bar{I}_{i, j}=E\left\{I_{i, j}\right\}$ is the local gray level expected value. The above values are calculated by using a weighted neighborhood $n_{i, j}$ centered about the actual pixel: 

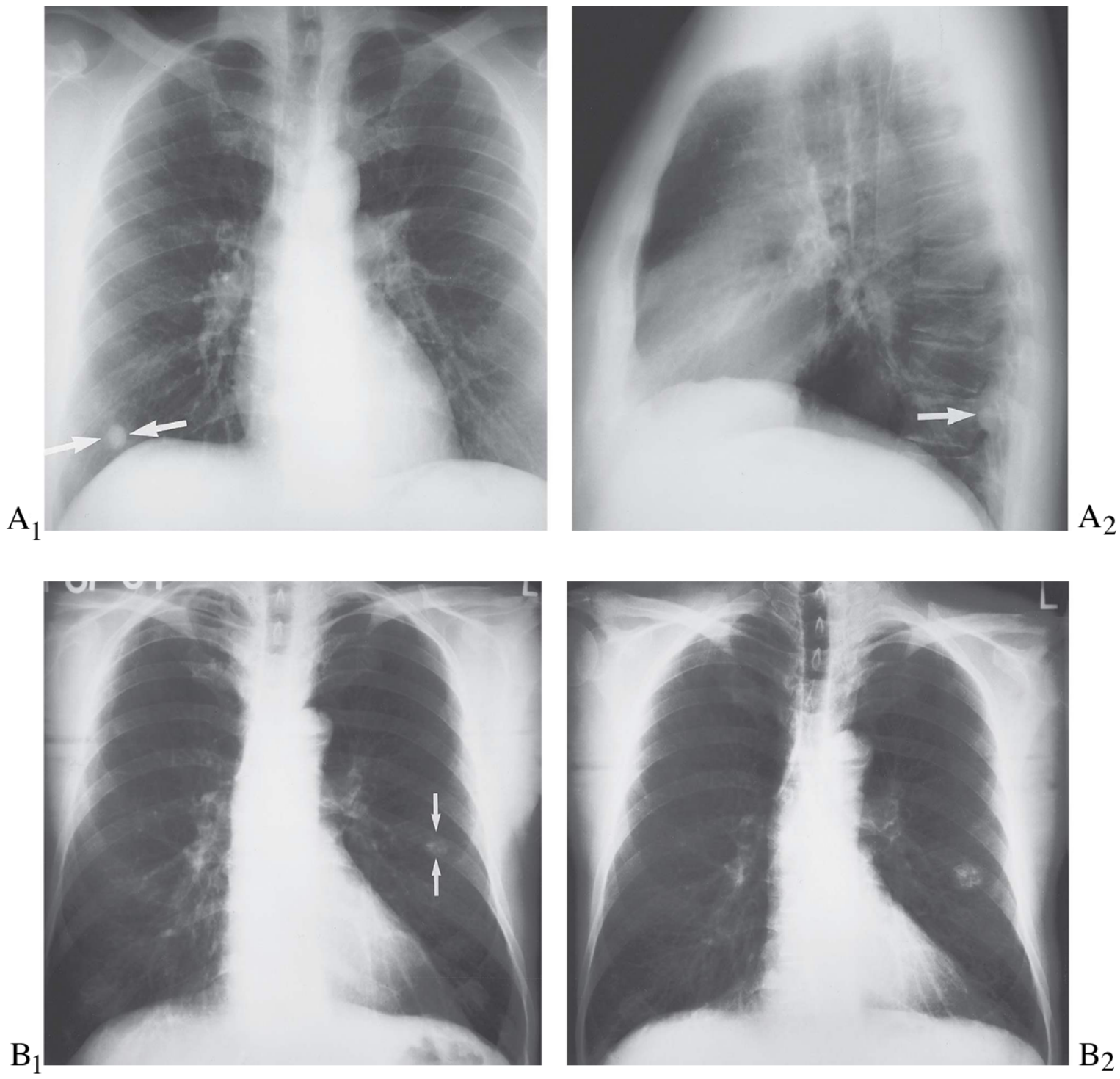

Fig. 1. Some samples of lung x-ray images, where pulmonary nodules were detected, taken from http://radiologykey.com. In $A_{1}$ is shown a posteroanterior chest radiograph where it was detected a small round opacity at the right lung base (arrows) due to the granuloma pathology. In $\mathrm{A}_{2}$ it is shown the lateral view of the opacity (arrow). The high density of the nodule and its small size indicates that it is highly calcified. The nodular density and its size is characteristic of a benign calcified granuloma. In $\mathrm{B}_{1}$ it is shown a posteroanterior chest radiograph where was detected a round calcified opacity in the left middle lung (arrows) due to the hamartoma pathology. While in $\mathrm{B}_{2}$ it is shown a posteroanterior chest radiograph obtained 5.5 years later. It is evident that the nodule size has increased (doubled in volume).

$$
\operatorname{var}\left(I_{i, j}\right)=\frac{\sum_{q \in n_{i, j}} w_{q}\left(I_{q}-\bar{I}_{i, j}\right)^{2}}{\sum_{q \in n_{i, j}} w_{q}}
$$

with

$$
\bar{I}_{i, j}=\frac{\sum_{q \in n_{i, j}} w_{q} I_{q}}{\sum_{q \in n_{i, j}} w_{q}}
$$

The size of the neighborhood $n_{i, j}$ should be related to the scale of the image structures expected for a particular application. The estimated local-variance of the image will be used as a quality measure of the structural similarity between two images. In fact, we will use some of its statistics. First, the mean of the local variance $\mu_{V}$ is calculated as:

$$
\mu_{V}=\frac{1}{M N} \sum_{i=1}^{M} \sum_{j=1}^{N} \operatorname{var}\left(I_{i, j}\right)
$$

Then the (global) standard deviation is defined as:

$$
\sigma_{V}=\left(E\left\{\left(\operatorname{var}\left(I_{i, j}\right)-\mu_{V}\right)^{2}\right\}\right)^{1 / 2}
$$

and it can be calculated as:

$$
\sigma_{V}=\left(\frac{1}{M N-1} \sum_{i=1}^{M} \sum_{j=1}^{N}\left(\operatorname{var}\left(I_{i, j}\right)-\mu_{V}\right)^{2}\right)^{1 / 2}
$$




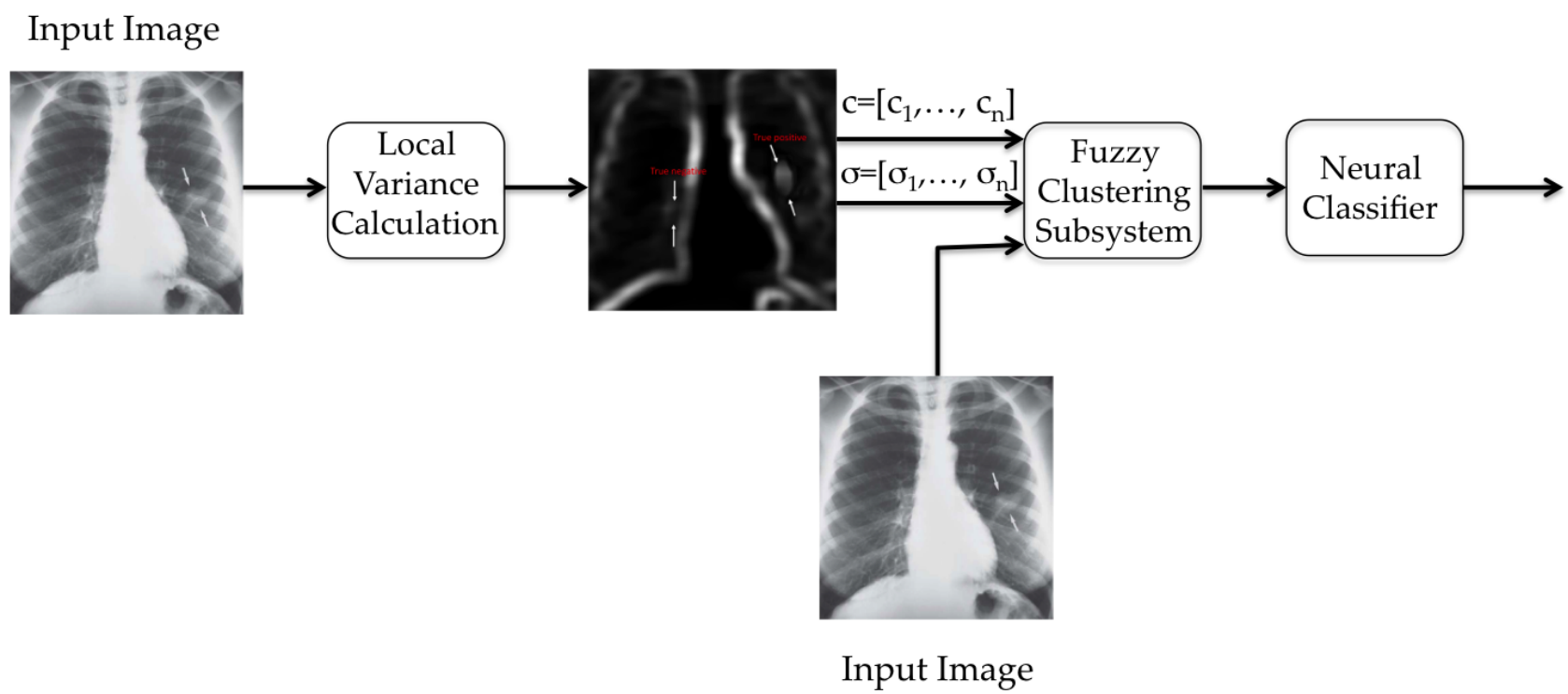

Fig. 2. Schematic description of the proposed procedure for lung nodules classification.

The most effective way to address these problems is that one of assuming that the image gray levels are piecewise constant or piecewise continuous. However, near the singular points, such as edges and textures, the assumption of being piecewise continuous and constant does not hold. It is well known from the literature that the context can be used to distinguish the singular points with respect to smooth points. Essentially, the context defined on the local gray level energy can be used for pixel classification. Then the use of a sliding window is necessary to discriminate pixels belonging to similar contexts.

The window size determines the nature of local energy in a gray level image, therefore when we use a sliding window to look for similarities it is essential to determine the correct window size, in order to ensure the reliability of estimates. Given the importance of the window size (neighborhood) in the local image processing, for its computation we proceeded as follows:

- First we calculate the mean value of the (global) standard deviation of the lung $\mathrm{x}$-ray database images coming from the dataset located at http://radiologykey.com by using the equation (6).

- Then through the equation (2) we calculate the mean value of the local variance for the nodules detected in the above dataset by the medical specialists.

More precisely:

$$
n=\frac{\sqrt{\operatorname{var}\left(I_{i, j}\right)}}{\sigma_{V}}
$$

Where $n \times n$ is the size of the sliding window. The obtained window size is $5 \times 5$.

Fig. 2 shows the main steps of the proposed procedure for lung nodules classification.

Finally, we implemented a maximum search algorithm by means of a directional mask [14] in order to find the local maxima within the variance matrix. This approach is inspired by our previous work [15].

Once these location have been obtained, lets define such locations as $c_{i}$ and the related local variance $\sigma_{i}$, the latters and the original $\mathrm{X}$-ray image are given as input to the fuzzy clustering system. Unfortunately the fuzzy system is not able to eliminate false positives, therefore, while extremely useful for a first screening step, it must be complemented by a more thorough classification system. This latter is provided by a probabilistic neural network. On the other hand, the neural network requires the data to be represented by means of a feature set, which, in this case, can be provided by means of the geometrical properties of the identified nodules. The adopted feature extraction technique is described in the following.

\section{A. Fuzzy logic-based segmentation}

The presented method consists in an unsupervised algorithm that arranges the data in clusters. In an unsupervised learning there are no labels that can be associated with the data to be classified and in the most general case the number of clusters present in the data is not known in advance. The clustering of data elements must be obtained on the basis of appropriate measures of similarity and distance defined with respect to the features that characterize the data [16]. More precisely, the clusters must be simultaneously homogeneous and well separated: the homogeneity implies that within the same cluster the elements must be resembling, while the separability imposes that elements belonging to different clusters must not resemble [17].

The standard approach to clustering generates partitions, so that, each element belongs to one and only one cluster. Instead the fuzzy clustering algorithms extend this notion by gradually attributing each element to each cluster through the use of an appropriate membership function. The lack of an objective and 
observable "ground truth" through which to verify the accuracy and reliability of the partitions obtained by the clustering algorithms, makes this learning problem substantially "illposed".

The unavailability of a priori knowledge about the classes to which the data belong requires an approach based on the analysis of the features extracted from the data in order to look for clues about the structures present in the data. On the other hand since the centroids supplied in input to the clustering system are in a number considerably higher than the possible nodules we have a high degree of uncertainty regarding the said search for structures in the data [18]. For this reason we decided to use a fuzzy approach to segmentation, because, as it is well known in the literature, the fuzzy logic allows an optimal management of uncertainty.

1) Input fuzzification: Since the characteristic parameters (e.g. pixel gray level, variance and so on) are numerical values, the membership can be obtained using the $\pi$ function (shown in Fig. 3). The definition of the $\pi$ function is:

$$
\pi(r, c, \sigma)=\exp \left(-\frac{\|r-c\|}{2 \sigma}\right)
$$

where $\sigma$ is the radius of the $\pi$ function and $c$ is the center point.

It follows that the membership functions are:

$$
\begin{aligned}
\mu_{i} & =\pi\left(r, c_{i}, \sigma_{i}\right) \\
\mu_{L_{i}} & =\pi\left(r, c_{L_{i}}, \sigma_{L_{i}}\right) \\
\mu_{h_{i}} & =\pi\left(r, c_{h_{i}}, \sigma_{h_{i}}\right) \\
\tilde{\mu}_{L_{i}} & =\pi\left(r, \tilde{c}_{L_{i}}, \sigma_{L_{i}}\right) \\
\tilde{\mu}_{h_{i}} & =\pi\left(r, \tilde{c}_{h_{i}}, \sigma_{h_{i}}\right)
\end{aligned}
$$

where

$$
\begin{gathered}
\sigma_{L_{i}}=\frac{\sigma_{i}}{s_{d}}, \quad \sigma_{h_{i}}=\frac{\sigma_{i}}{s_{d}}, \quad \tilde{c}_{i}=\left[c_{i x},-c_{i y}\right] \\
c_{L_{i}}=\left(1-\sigma_{L_{i}}\right) \cdot c_{i}, \quad \tilde{c}_{L_{i}}=\left(\sigma_{L_{i}}-1\right) \cdot c_{i} \\
c_{h_{i}}=\left(1+\sigma_{h_{i}}\right) \cdot \tilde{c}_{i}, \quad \tilde{c}_{h_{i}}=-\left(1+\sigma_{h_{i}}\right) \cdot \tilde{c}_{i}
\end{gathered}
$$

$s_{d}$ is the parameter of overlap-degree value that controls adjacent fuzzy sets. The overlap-degree parameter $s_{d}$ used in this example is 2 .

The adopted membership functions used impose a circular shape on the clusters, regardless of the effective data distribution, but since the centroids supplied in input to the clustering system are in a number considerably higher than the possible nodules some clusters will be partially overlapped. More precisely the clusters can be well-separated, continuously connected to each other, or overlapped each other, depending on the densities of the individual clusters and by the spatial relations and distances among the clusters.
2) Fuzzy model: The strategy with which the pixels are grouped in clusters identified by the centroids (clustering strategy) is now described in terms of human language and implemented by fuzzy rules.

The fuzzy rules has been written according to the following criteria:

- Distance from the pixel to the centroid $c_{i}$.

- Difference between highest and smallest gray level in the cluster.

- Maximum gradient of gray level of the pixels in the cluster.

- Minimum gradient of gray level of the pixels in the cluster.

The fuzzy sets structures are determined by fuzzy rules. The final crisp output values are calculated through the center of gravity method of defuzzification. The simulations are carried out by using the matlab toolbox. Gaussian-shaped membership functions were used for all the inputs feeding the fuzzy controller.

In Fig. 4 we can see some lung nodules extracted from the fuzzy clustering subsystem.

\section{B. Feature extraction based on geometric shape descriptors}

The feature extraction module evaluates for each object a set of parameters characterizing the features that are significant for the target object class. The lung nodules are uniquely identified by their local geometric structures. It follows that a simplified representation called "shape descriptor" turns out to be very effective. These simplified representations should retain most of the important information, while being easier to handle and to store. A complete shape descriptor is a representation that can be used to completely reconstruct the original object.

The features used in this paper are displayed in Tab. I Each object identified by previous stages is therefore represented, and output, by this module as a parameter set.

TABLE I

FEATURES USED FOR THE CLASSIFICATION OF THE LUNG NODULES EXTRACTED BY MEANS OF THE FUZZY MODULE.

\begin{tabular}{cc}
\hline Nomenclature & Feature \\
\hline$R$ & Area/perimeter ratio \\
$M E R$ & Minimum Rectangle Enclosing the nodule \\
$C$ & (perimeter) ${ }^{2} /$ area \\
$A R$ & Width/Length of $M E R$ \\
$R F F$ & (object's area) $/ M E R$ \\
\hline
\end{tabular}

\section{The implemented Probabilistic Neural Network}

In this work we used of probabilistic neural networks (PNN) for classification purposes. This kind of neural networks can be trained to identify whether or not a lung nodule constitutes a carcinoma.

PNNs have been succesfully implemented many times for classification in several field of applications [19]-[22].

In a PNN the input data, organized as an input vector, constitutes the input layer nodes. These data are fed into the first hidden layer, also called pattern layer, which is 

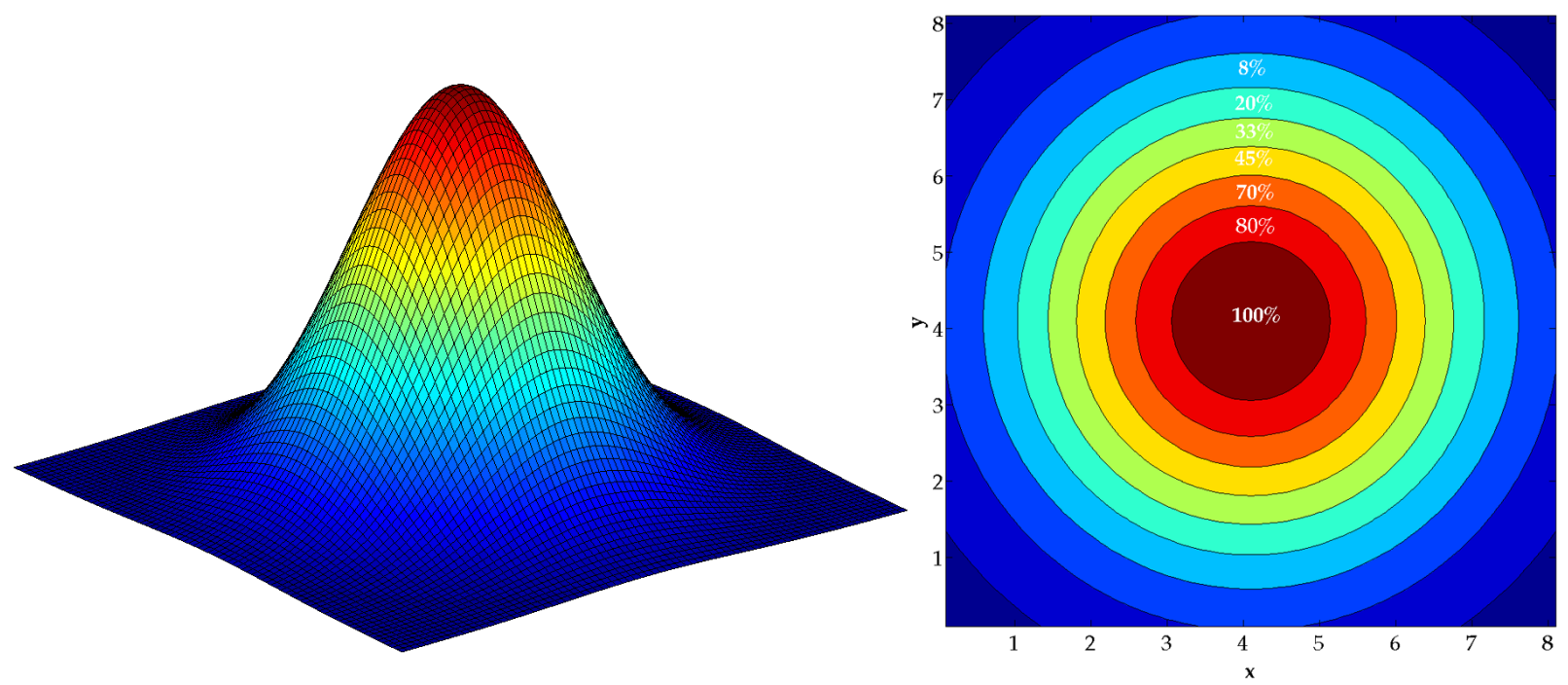

Fig. 3. In the left part of the picture is depicted the $\pi$ function used to obtain the membership functions, while in the right part of the picture is represented the membership degrees imposed by the $\pi$ function.
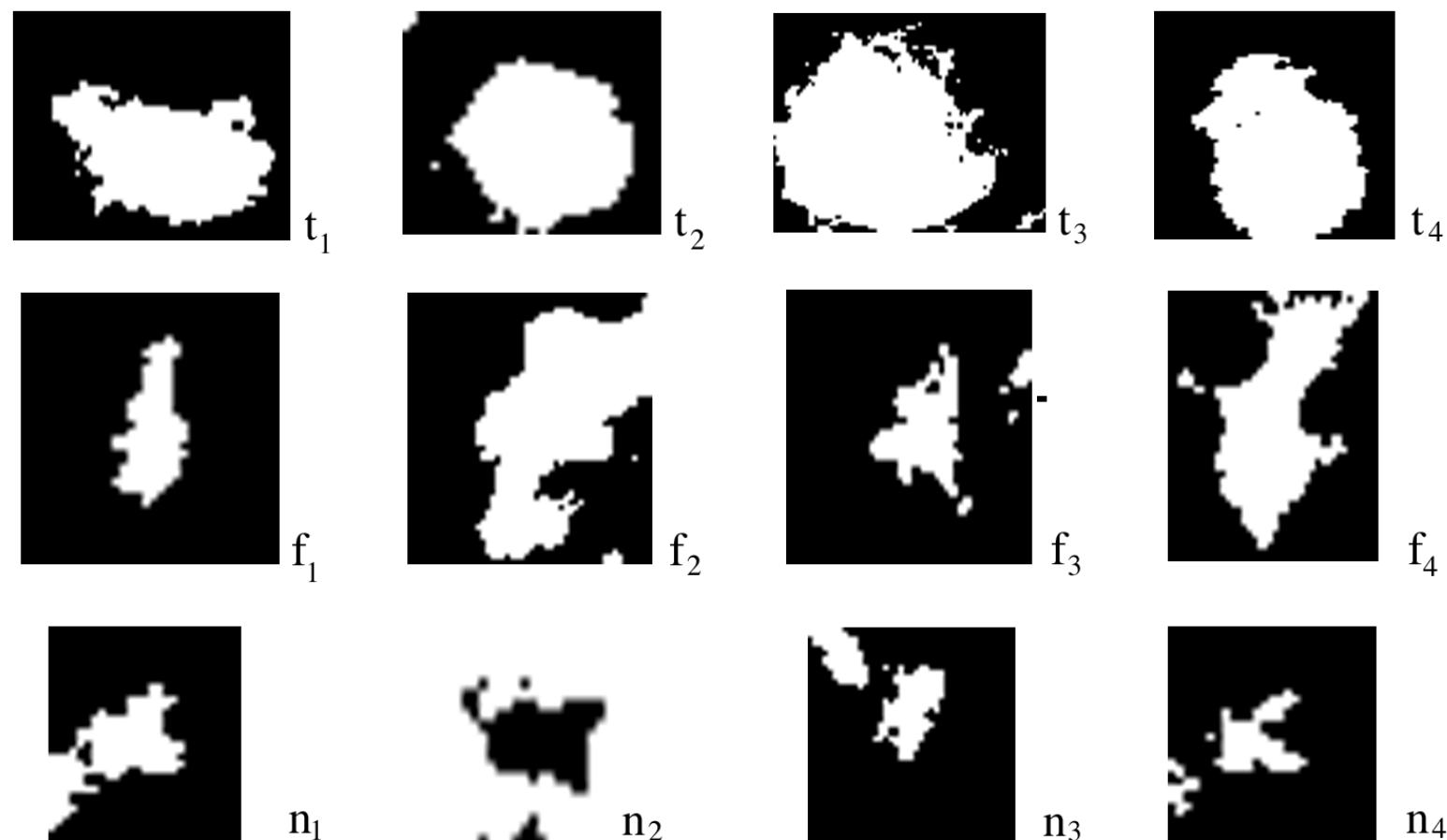

$\mathbf{n}_{1}$
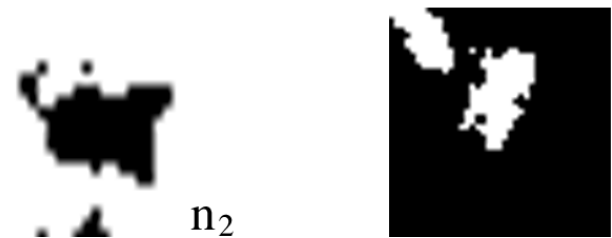

$\mathrm{n}_{3}$

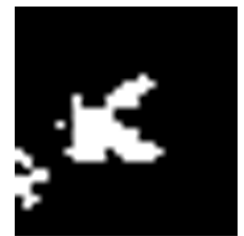

$\mathrm{n}_{4}$

Fig. 4. Some lung nodules extracted from the fuzzy clustering subsystem. In $t_{1}, t_{2}, t_{3}, t_{4}$ are shown examples of true nodules while in $f_{1}, f_{2}, f_{3}, f_{4}$ are shown examples of false nodules. In the subfigures $\mathrm{n}_{1}, \mathrm{n}_{2}, \mathrm{n}_{3}, \mathrm{n}_{4}$, some fake clusters extracted from the fuzzy clustering subsystem, are displayed.

composed by neurons activated by a Gaussian or Radial Basis function. The second hidden layer, or summation layer, is composed by neurons that performs an average operation on the outputs coming from the second layer. Each summation neuron represents a class or an option. Finally, the output layer selects the largest output coming from the summation layer by performing a vote and then determining the winning option, so that

$$
y_{k}(x(t))=\sum_{j=1}^{M} w_{k, j} \Phi_{j}(x(t))
$$

where

$$
\Phi_{j}(x(t))=\exp \left\{-\frac{1}{2 \sigma_{j}}\left(x(t)-\mu_{j}\right)^{T} \Sigma_{j}^{-1}\left(x(t)-\mu_{j}\right)\right\}
$$

where $\mu_{j}$ represents the elements of the mean vector an therefore the function centers represents the class' centroid, with $\Sigma_{j}$ the covariance matrix of the j-th basis, and with $\sigma_{j}$ defined as a smoothing parameter in order to control the basis function spread. 


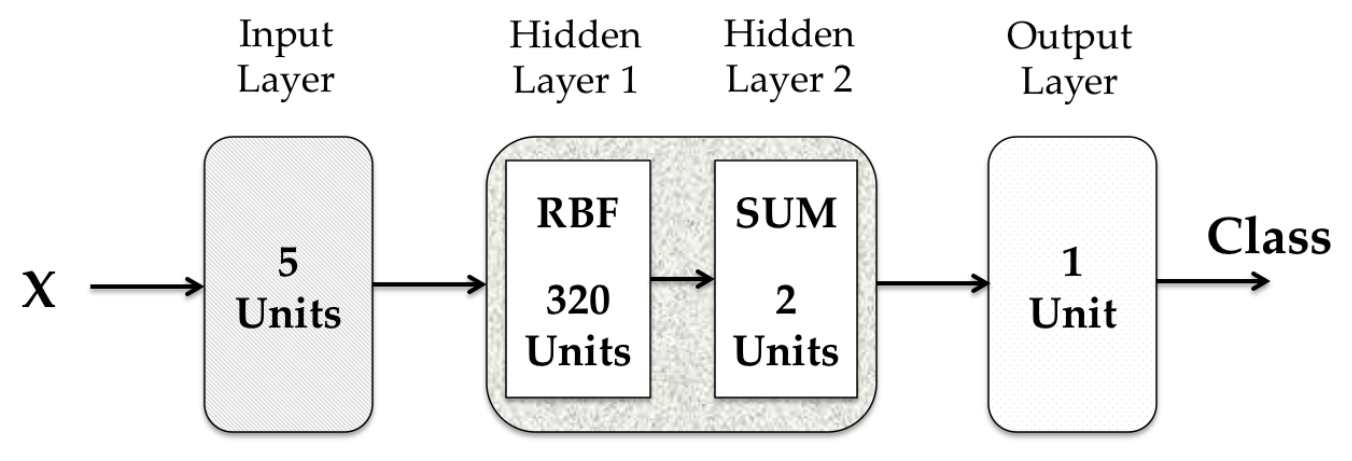

Fig. 5. The used probabilistic neural network. The components of the input vector $\mathbf{X}$ are the area/perimeter ratio of the nodule (R), the Minimum Rectangle Enclosing the nodule (MER), the square perimeter/area ratio of the nodule (C), the width/length of the nodule MER (AR), the area/MER of the nodule (RFF)

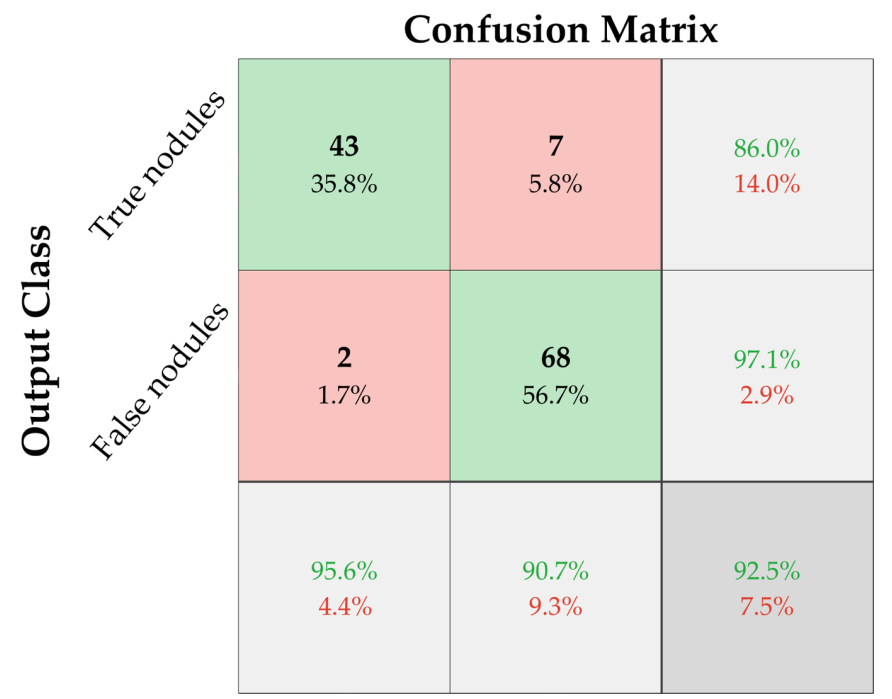

True nodules False nodules

\section{Target Class}

Fig. 6. Confusion matrix relative to the classification between true nodules and false nodules obtained by using the proposed method.

\section{Training of the PNN}

The training process for a PNN is composed of two stages: firstly the centers $c_{i}$ are found, after that the weights $w_{i, j}$ are computed.

The number of basis functions (neurons of the hidden layer) is typically much lower than the number of the training patterns. The centers of the basis functions don't necessarily have to belong to the set of training patterns, then the determination of the centers becomes part of the learning process.

In order to compute the weights, the PNN must be trained with supervised learning techniques. Instead, regarding the centers, there are two strategies:

1) Use an unsupervised learning to determine a set of bump locations $c_{i}$ and use LMS algorithm to train output weights $w_{i, j}$. This is a hybrid training scheme.

2) Use a second strategy that requires the training of all parameters: spread parameters $\sigma_{i}$ output weights $w_{i, j}$ and bump locations $c_{i}$ through a supervised learning algorithm (often the backpropagation algorithm).

Training methods that separate the tasks of prototype determination and weight optimization (the first strategy) often do not use the input-output data from the training set for the selection of the prototypes. For instance, the random selection method and the k-means algorithm result in prototypes that are completely independent of the input-output data from the training set. Although this results in fast training, it clearly does not take full advantage of the information contained in the training set. For this reason, in this paper, we have chosen the the second strategy by means of a backpropagation algorithm. In fact the gradient descent training of PNN networks has been proven to be much more effective than many conventional methods [23].

The Gaussian radial basis transfer function used for the neurons of the hidden layer is reported in the following:

$$
\phi(r)=e^{-(r / \sigma)^{2}}
$$

The radii $\sigma_{k}$ are initialized to equal values according to the heuristics

$$
\sigma_{k}=\frac{d_{\max }}{\sqrt{2 m}}
$$

where $d_{\max }$ is the maximum distance between the input vectors of two training patterns.

Then we have calculated the weights, the radii and center vectors by iteratively computing the partials derivates and performing the following updates: 


$$
\begin{gathered}
E=\frac{1}{2} \sum_{j=1}^{m}\left(d_{j}-y_{j}\right)^{2}=\frac{1}{2} \sum_{j=1}^{m} e_{j}^{2} \\
w_{i, j}^{\text {new }}=w_{i, j}^{\text {old }}-\eta_{1} \frac{\partial E}{\partial w_{i, j}} \\
c_{i}^{\text {new }}=c_{i}^{\text {old }}-\eta_{2} \frac{\partial E}{\partial c_{i}} \\
\sigma_{i}^{\text {new }}=\sigma_{i}^{\text {old }}-\eta_{3} \frac{\partial E}{\partial \sigma_{i}}
\end{gathered}
$$

where $\eta_{1}, \eta_{2}$ and $\eta_{3}$ are the learning rate coefficients. While the optimal number of neurons in the hidden layer has been established by the trial and error method.

The resulting network after the training phase [20] is shown in Fig. 5. It consists of 320 neurons. Once the optimal parameters are found the trained PNN as been used to classify the data points in the test set into one of the two classes.

The adopted neural network (Fig. 5) has five inputs: The area/perimeter ratio of the nodule (R), the Minimum Rectangle Enclosing the nodule (MER), the square perimeter/area ratio of the nodule $(\mathrm{C})$, the width/length of the nodule MER (AR), the area/MER of the nodule (RFF).

The neural network has one output that is used to classify the nodules into one of the two classes. The results show that the proposed approach provides an accuracy of $92.5 \%$ of correct classifications.

\section{BIO-INSPIRED REINFORCEMENT LEARNING}

Polarization and misleading dataset constitutes a paramount problem related to $\mathrm{x}$ ray imagery. Human anatomy can present variations related to the ethnic or geographic provenience of an individuals. On the other hand, the dataset used to train an automatic classification system are often collected by studies on a closed group of people (e.g. patients of the same hospital, collaborations within the same country, etc.).

It follows that the initial training set could lack generality, moreover can be subjected to possible, although improbable, mislabeling due to personal interpretation by the physician or relatively to the clinical history of the subject.

Moreover, while preserving its generality, the system at hand is required to minimize the risk of false negative results that, differently from a false positive diagnosis, could pose a threat for the health of a patient.

Fore these reasons in the presented work we devised a bioinspired reinforcement learning system in order to preserve the generalization capabilities of the probabilistic neural network.

Moreover the peculiar learning process adopted make it possible to take advantage from an increasing number of consultant and physicians, and therefore to partially integrate their diagnostic experience into the system. This also results in a noticeable speedup on the overall training time with a large increment of the eventual performances.

This bio-inspired training system has been inspired by the system has been inspired by the Hypothalamic-PituitaryThyroideal (HPT) model, shown in Fig. 7 which describes

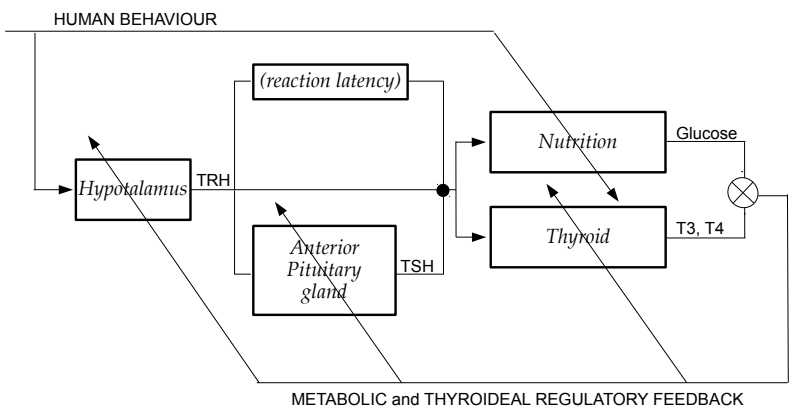

Fig. 7. The Hypothalamic-Pituitary-Thyroideal (HPT) model.

the self-regulatory mechanism of the human endocrine system. The HPT axis is responsible for regulating metabolism influencing food requirements and consequently the glucose level in human blood. The glands involved in the process are suggested by what HPT stands for, and they are: the hypothalamus, the anterior pituitary gland and the thyroid gland.

The hypothalamus triggers the release of the thyrotropinreleasing hormone (TRH) that increases the activity of the pituitary gland. The anterior pituitary gland (or adenohypophysis) leads the release or inhibition of a large number of neuroreceptors and hormones that are at the basis of a wide range of human reactions (as need for food, stress level, blood pressure etc.). The anterior pituitary gland can also release the thyroid-stimulating hormone (TSH) that directly increases the thyroidal functionalities. On the other hand, the thyroid gland produces thyroidal hormones regulating the metabolism, the intestinal and renal functions, and indirectly the glucose and brain functions by means of the hormones triiodothyronine (called T3) and tetraiodothyronine (called T4). The T3 hormone increases the basal metabolic rate and, thus, increases the body's oxygen and energy consumption, while the T4 increases the body's sensitivity to catecholamines (such as adrenaline) by permissiveness, which, on the long run, via some unknown mechanism, inhibits neuronal activity starting by the hypothalamus. Here the feedback closes, since the T3 and T4 hormones directly drive the hypothalamic functions inhibiting its capabilities to produce TRH. On the contrary, if the hypothalamus senses low circulating levels of thyroid hormone responds by releasing TRH and then stimulating the pituitary to produce TSH. In turn TSH-excited thyroid produces T3 until levels in the blood return to normal and by releasing $\mathrm{T} 4$ stops the hypothalamic functions exerting a negative feedback control. This negative feedback process is called thyroidal homeostasis and results from a multiloop feedback system that is found in virtually all higher vertebrates.

The thyroidal homeostasis and an appropriate regulation of the HPT axis is fundamental for the life of intelligent beings and their growth, differentiation, nutrition and social behaviour. Animals incapable of thyroideal homeostasis, and with a lesser-reacting HPT axis, exhibit a very low set-point that is assumed to underlie their metabolic and ontogenetic anomalies. 


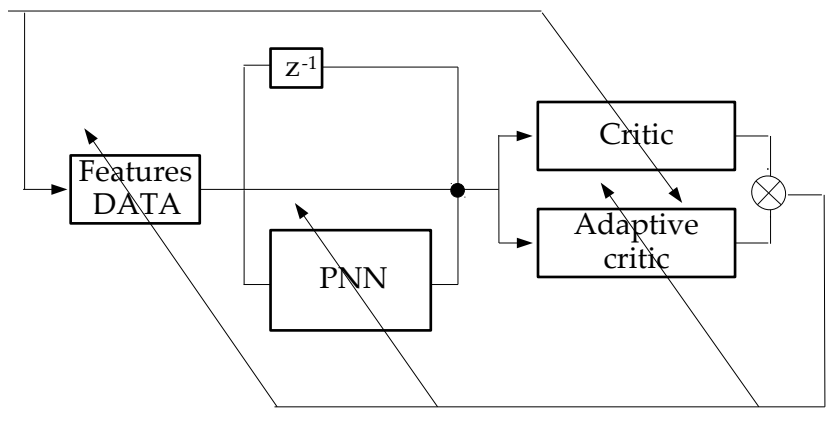

Fig. 8. Our classifier's continuous learning HPT-based feedback model mapping the human body features.

Taking into account the given similarities with the human physiological model, in this work we provided our PNN with additional modules enabling a continuous adaptive control for reinforcement learning.

Fig. 8 presents our applied solution, which is based on the HPT model, shown in Fig. 7

Since the field of application copes the developed system with the effective consequences of HPT decision, we used a supervised control configuration, where the external control is the result of choices and activities performed by the employer or manager. In our solution the PNN classifier is embedded into a larger modular system that controls the polarization attitude of the network and, when necessary, retrain it using new data collections.

To monitor the PNN polarization we implemented a modular architecture based on a critic decision component. This critic is initially a medical doctor or an operator which acts as an external agent on the system by evaluating eventual variation on the false positives (FP) or false negative (FN) ratio. When these measurement increases and the operator subjectively notice decreased performances a new training sequence can be triggered. During this phase software component, called adaptive critic, composed by a MLP network, models a soft threshold of uncertainty for the deviation of FN or FP, which in the long run should simulate the decision made by the human critic in order to diminish the human driven control over time. The adaptive critic learning is mediated by means of traditional gradient descent algorithm so that, called $\xi$ an error function, and $\Delta w_{i j}$ the weight modification is

$$
\Delta w_{i j}=-\mu \frac{\partial \xi}{\partial w_{i j}}=-\mu \frac{\partial \xi}{\partial \tilde{f}_{i}} \frac{\partial \tilde{f}_{i}}{\partial \tilde{u}_{i}} \frac{\partial \tilde{u}_{i}}{\partial w_{i j}}
$$

where $\tilde{f}_{i}$ is activation of $i$-esime neuron, $\mu$ the learning rate, and $\tilde{u}_{i}$ is the $i$-esime input to the neurone weighted as

$$
\tilde{u}_{i}=\sum_{j} w_{i j} \tilde{f}_{j}\left(\xi_{i}\right)
$$

The results of the adaptive control determines whether or not to train PNN with new and recently collected data.

\section{Results}

The proposed method was validated by means of a confusion matrix with Accuracy $(\Gamma)$, Sensitivity $(\chi)$ and Specificity $(\zeta)$ defined in classic way

$$
\begin{gathered}
\Gamma=\frac{T P+T N}{T P+T N+F P+F N}, \\
\chi=\frac{T P}{T P+F N}, \\
\zeta=\frac{T N}{T N+F P} .
\end{gathered}
$$

Where TP, FP, TN and FN represent respectively the true positives, false positives, true negatives and false negatives lung nodules.

The measure of Sensitivity reflects correct a classification of true positive samples, while the measure of Specificity reflects a correct classification of true negative samples. The measure of Accuracy reflects the correctness of sample classification between classes. Tab. II reports the results of our method with respect to other approaches. In our experiments we used 100 images. From these images the fuzzy clustering subsystem has extracted 440 possible nodules. We have used 320 of them for the training of the PNN while the remaining ones for the testing of the neural network. The results show the effectiveness of the proposed methodology. In fact we obtained correct classifications for $92.56 \%$ of samples within the testing data set.

In Fig. 6 we see a confusion matrix. In our experiments we have used x-ray images from participating clinic and open data set radiologykey.com. The dataset was composed by images that come from the open data set radiologykey.com and from clinical trials of nodules in lungs from Zagłębiowskie Oncology Centre, Poland.

Tab. [I] reports other methods used in classification of lung nodules. Most of classifiers uses Support Vector Machine or Neural Networks with various feature extraction. Our classifier used fuzzy model to extract clusters of pixels with potential defects. Most of the reported approaches uses. Accuracy measure as a main evaluation method.

Few methods could look like outperforming the proposed technique if we evaluate them only in terms of accuracy, sensitivity or specificity. On the other hand, analyzing the ensemble of all these three measurements, and particularly taking into account the overall effect between sensitivity and specificity, our technique shows the best performances. In facts, while the the accuracy of the proposed technique is $92.56 \%$, and its specificity is $90.79 \%$, the sensitivity is $95.56 \%$. Moreover, simplicity and self sustainability are paramount aspects of our technique that should be considered. Therefore our proposed approach results in improved performances while being implementable by means of smaller training sets. In facts while in our experiments we were able to reach similar results with respect to a standard training procedure, the implemented system required a $20 \%$ smaller training dataset with a training time reduction up to $25 \%$.

\section{Discussion}

The X-ray imagery for chest's area examination is commonly used in pulmonary clinics. However this type of exams 
TABLE II

LUNG NODULES DETECTION IN VARIOUS RESEARCH. DIFFERENT CLASSIFICATION APPROACHES FOR LUNG NODULES.

\begin{tabular}{|c|c|c|c|c|c|}
\hline Method & Features & Classifier & Accuracy & Sensitivity & Specificity \\
\hline Wei et al. 25 & texture and learned distance metric & $\begin{array}{l}\text { Two Step Content-Based Image } \\
\text { Retrieval (TSCBIR) }\end{array}$ & $91 \%$ & NA & NA \\
\hline Yuan et al. [12] & hybrid descriptors & $\begin{array}{l}\text { multi-class Support Vector Ma- } \\
\text { chine (SVM) }\end{array}$ & $93.9 \%$ & NA & NA \\
\hline Zhang et al. [13] & Common Spatial Pattern (CSP) & $\begin{array}{l}\text { Multi-kernel Extreme Learning } \\
\text { Machine (ELM) }\end{array}$ & $87.5 \%$ & NA & NA \\
\hline Karargyris et al. [27] & texture and shape comparison & Support Vector Machine (SVM) & NA & $92.1 \%$ & $84 \%$ \\
\hline Santosh et al. [28] & edge map and histograms & $\mathrm{NN}$ & $86.36 \%$ & NA & NA \\
\hline Maduskar et al. 29] & $\begin{array}{l}\text { landmark structure and segmenta- } \\
\text { tion }\end{array}$ & k Nearest Neighbors (kNN) & NA & $92 \%$ & $70 \%$ \\
\hline Zhang et al. [30] & Laplace priors & SBLaplace & $95 \%$ & NA & NA \\
\hline Demir and Camurcu [35] & rules & Support Vector Machine (SVM) & $88.97 \%$ & $98.03 \%$ & $87.71 \%$ \\
\hline Jacobs et al. 36 & NA & Computer Aided Detection (CAD) & $82 \%$ & $83 \%$ & NA \\
\hline Han et al. $|37|$ & vector quantization & Support Vector Machine (SVM) & NA & $92.7 \%$ & $93.3 \%$ \\
\hline Akram et al. 38 & multiple thresholding & Support Vector Machine (SVM) & NA & $96.31 \%$ & $96.77 \%$ \\
\hline Wang et al. 39 & 3D detection & Support Vector Machine (SVM) & NA & $93 \%$ & NA \\
\hline Proposed method (Neuro-fuzzy) & geometric shape descriptors & PNN & $92.56 \%$ & $95.56 \%$ & $90.79 \%$ \\
\hline
\end{tabular}

has many limitations, since a variety of pathological changes in the lung tissues could result in many similarities to unsospicious phisiological items visible in the screening. Many approaches are attempting to solve these problems, like this in [40], by analizing public data from web services.

Algorithms in literature mostly regards consecutive stages in image processing used in order to find a search space in which demarcation of tissues can be done by means of extraction methods. After that, following the common workflow, the nodules are extracted and false candidates are preliminary eliminated from further research. Finally the main classifiers work on the samples to evaluate them and find true positives. In Tab. II we can see a summary of methods applied to lung cancer detection.

In [41] it was used a combination of first and second order probabilistic descriptors to smoothen Gaussian filtered images. The latter, then were used for segmentation of lungs pathologic tissues from 3D images. Three step segmentation was proposed in [42]. After Hessian analysis of tub-like structures a multi cavity enhancement filter identified potential cancer tissues. In the final stage a Support Vector Machine was used to evaluate the images and eliminate false positive regions. Deep Convolutional Neural Networks were used in [43] for detection of cancer using chest X-ray images. Experiments were done over 504 images of two-exposure dual-energy subtraction x-ray screenings of the chest (404 in training and 100 in test). A similar approach with wide comparisons to other method was discussed in [44]. Presented approaches are based on machine learning, however most of them are not able to classify low-contrast nodules with $20 \mathrm{~mm}$ diameter and low density. Therefore in this paper we present a new classification method for lung carcinomas based on fuzzy logic used for segmentation of clusters with potential lung tumors. In the second stage we applied Probabilistic Neural Network as classifier for these clusters. The proposed method is able to detect not only low-contrast nodules but also lung cancers of minor or equal to $20 \mathrm{~mm}$ diameter.

\section{Conclusions}

The simplicity of the proposed method is one of the main advantages. Moreover it has obtained very good results. In comparison to other approaches presented in Tab. II we were able to achieve: better or equal correct classification rate $(92.56 \%)$ and ability to detect low-contrast nodules.

Moreover we believe that such a diagnostic support should firstly focus on the sensitivity of the approach due to the necessity to minimize the false negative ratio. In fact while false positive could lead to unnecessary further examinations, a false negative could lead to a dangerous and potentially lethal misdiagnosis. In Tab. II the approaches that offers a specificity greater than $90 \%$ are highlighted in bold, while, among these latter, the approaches with a sensitivity greater than $90 \%$ are highlighted in italic. While we consider an high specificity a priority, we also consider very important an high sensitivity. This latter is a mandatory requirement for a practically applicable diagnostic tool. While in literature only three approaches ( [11], [38], and [39]) meet the sensitivity requirement, only two of them ( [38] and [39]) also meet the specificity criterion. It is also possible to see that our approach equals or outperforms the sensitivity and specificity of other approaches in literature while preserving an high accuracy $(92.56 \%)$. 
In further research we want to apply this methodology to other pulmonary diseases, which have similar symptoms. From our early research we can conclude that this kind of approach can be easily adjusted to other requirements due to the use of fuzzy rules without any loss in precision.

\section{REFERENCES}

[1] O. M. Mets, K. Chung, E. T. Scholten, W. B. Veldhuis, M. Prokop, B. van Ginneken, C. M. Schaefer-Prokop, and P. A. de Jong, "Incidental perifissural nodules on routine chest computed tomography: lung cancer or not?" European radiology, vol. 28, no. 3, pp. 1095-1101, 2018.

[2] R. Yip, K. Li, L. Liu, D. Xu, K. Tam, D. F. Yankelevitz, E. Taioli, B. Becker, and C. I. Henschke, "Controversies on lung cancers manifesting as part-solid nodules," European radiology, vol. 28, no. 2, pp. 747-759, 2018.

[3] C.-H. Chen, C.-K. Chang, C.-Y. Tu, W.-C. Liao, B.-R. Wu, K.-T. Chou, Y.-R. Chiou, S.-N. Yang, G. Zhang, and T.-C. Huang, "Radiomic features analysis in computed tomography images of lung nodule classification," PloS one, vol. 13, no. 2, p. e0192002, 2018.

[4] D. Mousa, N. Zayed, and M. Fakhr, "Significant features to detect pulmonary nodules from ct lung images," Biomedical Engineering: Applications, Basis and Communications, vol. 29, no. 06, p. 1750045, 2017.

[5] S. Naqi, M. Sharif, M. Yasmin, and S. L. Fernandes, "Lung nodule detection using polygon approximation and hybrid features from ct images," Current Medical Imaging Reviews, vol. 14, no. 1, pp. 108117, 2018.

[6] P. Melo-Pinto, P. Couto, H. Bustince, E. Barrenechea, M. Pagola, and J. Fernandez, "Image segmentation using atanassov's intuitionistic fuzzy sets," Expert Systems with Applications, vol. 40, no. 1, pp. 15-26, 2013.

[7] A. Bigand and O. Colot, "Membership function construction for intervalvalued fuzzy sets with application to gaussian noise reduction," Fuzzy Sets and Systems, vol. 286, pp. 66-85, 2016.

[8] O. Uncu and I. B. Turksen, "Discrete interval type 2 fuzzy system models using uncertainty in learning parameters," IEEE Transactions on Fuzzy Systems, vol. 15, no. 1, pp. 90-106, 2007.

[9] H. Bustince, E. Barrenechea, M. Pagola, J. Fernández, and J. Sanz, "Comment on: "image thresholding using type ii fuzzy sets". importance of this method," Pattern Recognition, vol. 43, no. 9, pp. 3188-3192, 2010.

[10] H. Bustince, M. Pagola, E. Barrenechea, J. Fernández, P. Melo-Pinto, P. Couto, H. R. Tizhoosh, and J. Montero, "Ignorance functions. an application to the calculation of the threshold in prostate ultrasound images," Fuzzy sets and Systems, vol. 161, no. 1, pp. 20-36, 2010.

[11] Y. Xie, J. Zhang, Y. Xia, M. Fulham, and Y. Zhang, "Fusing texture, shape and deep model-learned information at decision level for automated classification of lung nodules on chest ct," Information Fusion, vol. 42, pp. 102-110, 2018

[12] J. Yuan, X. Liu, F. Hou, H. Qin, and A. Hao, "Hybrid-feature-guided lung nodule type classification on ct images," Computers \& Graphics, vol. 70 , pp. 288-299, 2018

[13] Y. Zhang, Y. Wang, G. Zhou, J. Jin, B. Wang, X. Wang, and A. Cichocki, "Multi-kernel extreme learning machine for eeg classification in braincomputer interfaces," Expert Systems with Applications, vol. 96, pp. 302-310, 2018

[14] S. K. Vipparthi, S. Murala, A. B. Gonde, and Q. M. J. Wu, "Local directional mask maximum edge patterns for image retrieval and face recognition," IET Computer Vision, vol. 10, no. 3, pp. 182-192, 2016, DOI: 10.1049/iet-cvi.2015.0035.

[15] M. Woźniak, D. Połap, G. Capizzi, G. Lo Sciuto, L. Kośmider, and K. Frankiewicz, "Small lung nodules detection based on local variance analysis and probabilistic neural network," Computer methods and programs in biomedicine, vol. 161, pp. 173-80, 2018.

[16] M. B. Gorzałczany and F. Rudziński, "Generalized self-organizing maps for automatic determination of the number of clusters and their multiprototypes in cluster analysis," IEEE Transactions on Neural Networks and Learning Systems, vol. 29, no. 7, pp. 2833-2845, July 2018.

[17] S. Ben-David and U. Von Luxburg, "Relating clustering stability to properties of cluster boundaries." in COLT, vol. 2008, 2008, pp. 379390.

[18] Y. Lin, S. Liao, J. Chang, and C. Lin, "Simplified interval type-2 fuzzy neural networks," IEEE Transactions on Neural Networks and Learning Systems, vol. 25, no. 5, pp. 959-969, May 2014.
[19] S. N. Barnea, G. Lo Sciuto, N. Hai, R. Shikler, G. Capizzi, M. Woźniak, and D. Połap, Photo-Electro Characterization and Modeling of Organic Light-Emitting Diodes by Using a Radial Basis Neural Network. Cham: Springer International Publishing, 2017, pp. 378-389.

[20] F. Beritelli, G. Capizzi, G. Lo Sciuto, C. Napoli, and F. Scaglione, "Automatic heart activity diagnosis based on gram polynomials and probabilistic neural networks," Biomedical Engineering Letters, Aug 2017.

[21] F. Beritelli, G. Capizzi, G. Lo Sciuto, F. Scaglione, D. Połap, and M. Woźniak, A Neural Network Pattern Recognition Approach to Automatic Rainfall Classification by Using Signal Strength in LTE/4G Networks. Cham: Springer International Publishing, 2017, pp. 505-512.

[22] A. Alexandridis, E. Chondrodima, N. Giannopoulos, and H. Sarimveis, "A fast and efficient method for training categorical radial basis function networks," IEEE Transactions on Neural Networks and Learning Systems, vol. 28, no. 11, pp. 2831-2836, Nov 2017.

[23] N. B. Karayiannis and G. W. Mi, "Growing radial basis neural networks: merging supervised and unsupervised learning with network growth techniques," IEEE Transactions on Neural Networks, vol. 8, no. 6, pp. 1492-1506, 1997.

[24] B. N. Narayanan, R. C. Hardie, and T. M. Kebede, "Performance analysis of a computer-aided detection system for lung nodules in ct at different slice thicknesses," Journal of Medical Imaging, vol. 5, no. 1, p. $014504,2018$.

[25] G. Wei, H. Cao, H. Ma, S. Qi, W. Qian, and Z. Ma, "Content-based image retrieval for lung nodule classification using texture features and learned distance metric," Journal of medical systems, vol. 42, no. 1, p. 13, 2018 .

[26] Y. Jiao, Y. Zhang, Y. Wang, B. Wang, J. Jin, and X. Wang, "A novel multilayer correlation maximization model for improving cca-based frequency recognition in ssvep brain-computer interface," International journal of neural systems, p. 1750039, 2017.

[27] A. Karargyris, J. Siegelman, D. Tzortzis, S. Jaeger, S. Candemir, Z. Xue, K. C. Santosh, S. Vajda, S. K. Antani, L. Folio, and G. R. Thoma, "Combination of texture and shape features to detect pulmonary abnormalities in digital chest x-rays," Int. J. Computer Assisted Radiology and Surgery, vol. 11, no. 1, pp. 99-106, 2016, DOI: 10.1007/s11548-015-1242-x.

[28] K. C. Santosh, S. Vajda, S. K. Antani, and G. R. Thoma, "Edge map analysis in chest $\mathrm{X}$-rays for automatic pulmonary abnormality screening," Int. J. Computer Assisted Radiology and Surgery, vol. 11, no. 9, pp. 1637-1646, 2016, DOI: 10.1007/s11548-016-1359-6.

[29] P. Maduskar, R. H. H. M. Philipsen, J. Melendez, E. T. Scholten, D. Chanda, H. Ayles, C. I. Sánchez, and B. van Ginneken, "Automatic detection of pleural effusion in chest radiographs," Medical Image Analysis, vol. 28, pp. 22-32, 2016, DOI: 10.1016/j.media.2015.09.004.

[30] Y. Zhang, G. Zhou, J. Jin, Q. Zhao, X. Wang, and A. Cichocki, "Sparse bayesian classification of eeg for brain-computer interface," IEEE transactions on neural networks and learning systems, vol. 27, no. 11, pp. 2256-2267, 2016.

[31] A. K. Dhara, S. Mukhopadhyay, A. Dutta, M. Garg, and N. Khandelwal, "A combination of shape and texture features for classification of pulmonary nodules in lung ct images," Journal of digital imaging, vol. 29 , no. 4, pp. 466-475, 2016

[32] A. A. A. Setio, F. Ciompi, G. Litjens, P. Gerke, C. Jacobs, S. J. van Riel, M. M. W. Wille, M. Naqibullah, C. I. Sánchez, and B. van Ginneken, "Pulmonary nodule detection in ct images: false positive reduction using multi-view convolutional networks," IEEE transactions on medical imaging, vol. 35, no. 5, pp. 1160-1169, 2016.

[33] H. M. Orozco, O. O. V. Villegas, V. G. C. Sánchez, H. d. J. O. Domínguez, and M. d. J. N. Alfaro, "Automated system for lung nodules classification based on wavelet feature descriptor and support vector machine," Biomedical engineering online, vol. 14, no. 1, p. 9, 2015.

[34] J.-Y. Zeng, H.-H. Ye, S.-X. Yang, R.-C. Jin, Q.-L. Huang, Y.-C. Wei, S.-G. Huang, B.-Q. Wang, J.-Z. Ye, and J.-Y. Qin, "Clinical application of a novel computer-aided detection system based on three-dimensional ct images on pulmonary nodule," International journal of clinical and experimental medicine, vol. 8, no. 9, p. 16077, 2015.

[35] Ö. Demir and A. Yılmaz Çamurcu, "Computer-aided detection of lung nodules using outer surface features," Bio-medical materials and engineering, vol. 26, no. s1, pp. S1213-S1222, 2015.

[36] C. Jacobs, E. M. van Rikxoort, K. Murphy, M. Prokop, C. M. SchaeferProkop, and B. van Ginneken, "Computer-aided detection of pulmonary nodules: a comparative study using the public lidc/idri database," European radiology, vol. 26, no. 7, pp. 2139-2147, 2016.

[37] H. Han, L. Li, F. Han, B. Song, W. Moore, and Z. Liang, "Fast and adaptive detection of pulmonary nodules in thoracic ct images using a 
hierarchical vector quantization scheme," IEEE journal of biomedical and health informatics, vol. 19, no. 2, pp. 648-659, 2015.

[38] S. Akram, M. Y. Javed, A. Hussain, F. Riaz, and M. Usman Akram, "Intensity-based statistical features for classification of lungs ct scan nodules using artificial intelligence techniques," Journal of Experimental \& Theoretical Artificial Intelligence, vol. 27, no. 6, pp. 737-751, 2015.

[39] Q. Wang, W. Zhu, and B. Wang, "Three-dimensional svm with latent variable: application for detection of lung lesions in ct images," Journal of medical systems, vol. 39, no. 1, p. 171, 2015.

[40] S. Khasnavis, A. B. Rosenkrantz, and V. Prabhu, "Using twitter to assess the public response to the united states preventive services task force guidelines on lung cancer screening with low dose chest CT," J. Digital Imaging, vol. 30, no. 3, pp. 323-327, 2017, DOI: 10.1007/s10278-0169933-6.

[41] A. Soliman, F. Khalifa, A. Elnakib, M. A. El-Ghar, N. Dunlap, B. Wang, G. L. Gimel'farb, R. Keynton, and A. El-Baz, "Accurate lungs segmentation on CT chest images by adaptive appearance-guided shape modeling," IEEE Trans. Med. Imaging, vol. 36, no. 1, pp. 263-276, 2017, DOI: 10.1109/TMI.2016.2606370.

[42] Q. Meng, T. Kitasaka, Y. Nimura, M. Oda, J. Ueno, and K. Mori, "Automatic segmentation of airway tree based on local intensity filter and machine learning technique in 3d chest CT volume," Int. J. Computer Assisted Radiology and Surgery, vol. 12, no. 2, pp. 245-261, 2017, DOI: 10.1007/s11548-016-1492-2.

[43] W. Yang, Y. Chen, Y. Liu, L. Zhong, G. Qin, Z. Lu, Q. Feng, and W. Chen, "Cascade of multi-scale convolutional neural networks for bone suppression of chest radiographs in gradient domain," Medical Image Analysis, vol. 35, pp. 421-433, 2017, DOI: 10.1016/j.media.2016.08.004.

[44] Y. Xiao, J. Wu, Z. Lin, and X. Zhao, "A deep learning-based multi-model ensemble method for cancer prediction," Computer Methods and Programs in Biomedicine, vol. 153, pp. 1-9, 2018, DOI: 10.1016/j.cmpb.2017.09.005.

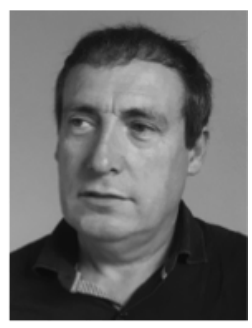

GIACOMO CAPIZZI received the Laurea degree (summa cum laude) in electronic engineering from the University of Catania, Catania, Italy, in 1993, and the Ph.D. degree in electronic and computer engineering from the University of Reggio Calabria, Reggio Calabria, Italy, in 2000. From 1993 to 1996, he was a Cabling Systems Designer with Itel (a small company of telecommunications). From 2000 to 2002, he was a Lecturer with the Department of Electrical, Electronics and System Engineering, University of Catania, where he joined as an Assistant Professor in 2002. From 2015 to 2016, 2016 to 2017, and from 2017 to 2018, he was an Invited Professor with the Silesian University of Technology to teach in a master course on the topic algorithms and paradigms for pattern recognition (course that currently teaches). His research interests include wavelet theory, neural networks, statistical pattern recognition, Bayesian networks, theory and design of linear and nonlinear digital/analog filters, integrated generation systems, renewable energy sources, and battery storage modeling and simulation.

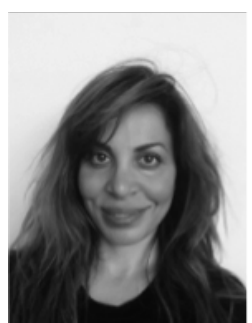

GRAZIA LO SCIUTO received the Ph.D. degree in applied electronics from the University of Rome Tre in 2016. In 2015, she received the scholarship on the optical calculations for large-scale organic photovoltaic with ENEA-BGU Joint Laboratory, Department of Electrical and Computer Engineering, Ben-Gurion University of the Negev, Israel. Her research interests include electronic devices, semiconducting polymers, organic materials, novel devices for photovoltaic, and neural networks applied to complex systems, such as renewable energy, signal processing, pattern recognition, and biometrics.

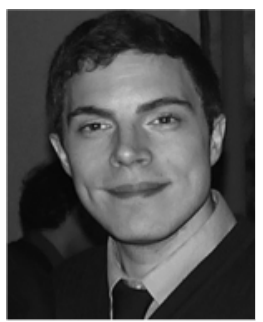

CHRISTIAN NAPOLI is Associate Professor with the Department of Computer, Control, and Management Engineering "Antonio Ruberti", Sapienza University of Rome, since 2019, as well as Scientific Director and co-founder (2015) of the International School of Advanced and Applied Computing (ISAAC). He received the B.Sc. degree in Physics from the Department of Physics and Astronomy, University of Catania, in 2010, where he also got the M.Sc. degree in Astrophysics in 2012 and the Ph.D. in Computer Science in 2016 at the Department of Mathematics and Computer Science. He has been Research Associate with the Department of Mathematics and Computer Science, University of Catania, from 2018 to 2019, while, previously, Research Fellow and Adjunct Professor with the same department from 2015 to 2018. He has been several time Invited Professor at the Silesian University of Technology, Visiting Academic at the New York University. His teaching activity focused on Artificial Intelligence, Neural Networks, Machine Learning, Computing Systems, Computer Architectures, Distributed Systems, and High Performance Computing. His current research interests include neural networks, artificial intelligence, computational models, and high performance computing.

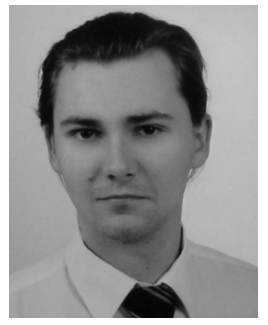

DAWID POŁAP received diplomas in Computer Science and Applied Mathematics (with honors), in 2015 and 2017 receptively, and $\mathrm{PhD}$ in 2019. $\mathrm{He}$ is an adjunct at the Faculty of Applied Mathematics of the Silesian University of Technology in Gliwice, Poland. His main scientific interests are image processing and various aspects of machine learning. In his career, he was awarded by the Polish Ministry of Science and Higher Education with a "Diamond Grant" for the most talented students. Dawid Połap was participating in various scientific projects (both as lead investigator and scientific investigator) from polish and italian universities. He has published above fifty research papers in international conferences and journals. Dawid Połap served as an editor for various special issues of Algorithms, etc.

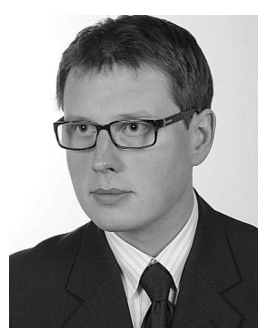

MARCIN WOŹNIAK received diplomas in Applied Mathematics and Computational Intelligence, $\mathrm{PhD}$ in 2012 and DSc in 2019. He is an Assoc. Professor at the Faculty of Applied Mathematics of the Silesian University of Technology in Gliwice, Poland. His main scientific interests are neural networks with their applications together with various aspects of applied computational intelligence. He is a scientific supervisor in editions of "the Diamond Grant" and "the Best of the Best" programs for highly talented students from the Polish Ministry of Science and Higher Education. He participated in various scientific projects (as lead investigator and scientific investigator or participant) at polish and italian universities. In scientific career he was visiting researcher at universities in Italy, Sweden and Germany. He has published around one hundred research papers in international conferences and journals. Marcin Woźniak served as an editor for various special issues of Sensors, IEEE ACCESS, CIN, etc., and as an organizer or a session chair at various international conferences and symposiums like IEEE SSCI, etc. 